\title{
ARTICLE OPEN Mechanics of thermally fluctuating membranes
}

\author{
J. H. Los ${ }^{1}$, A. Fasolino (D) and M. I. Katsnelson ${ }^{1}$
}

Besides having unique electronic properties, graphene is claimed to be the strongest material in nature due to its Young modulus, which is, per atomic layer, much larger than that of steel. This reasoning however does not take into account the peculiar properties of graphene as a thermally fluctuating crystalline membrane, which at finite temperature, lead to a dramatic reduction of the Young modulus for micron-sized graphene samples in comparison with atomic scale values. We show that the standard Föppl-von Karman elasticity theory for thin plates, routinely used for the interpretation of experimental results has to be modified for graphene at room temperature and for micron-sized samples. Based on scaling analysis and atomistic simulation, we investigate the mechanics of graphene under transverse load up to breaking. We determine the limits of applicability of the Föppl-von Karman theory and provide quantitative estimates for the different regimes.

npj 2D Materials and Applications (2017)1:9; doi:10.1038/s41699-017-0009-3

\section{INTRODUCTION}

Graphene is claimed to be the strongest material in nature, ${ }^{1,2}$ with a Young modulus which is, per atomic layer, much larger than that of steel. In the press release of the Nobel committee (https://www. nobelprize.org/nobel_prizes/physics/laureates/2010/advancedphysicsprize2010.pdf), it is claimed that a hammock made of a squared meter of one-atom-thick graphene could sustain the weight of a $4 \mathrm{~kg}$ cat. More practically important are many applications of graphene-like scaffolds ${ }^{3}$ and sensors, ${ }^{4}$ which are crucially dependent on the mechanical strength. Meter-sized graphene is even being considered as a material for the lightsails in the starshot project to reach the star alpha centaury (https:// breakthroughinitiatives.org/Initiative/3). The predicted exceptional strength of graphene however is based on the conventional theory of thin plates, ${ }^{5,6}$ which does not take into account that graphene has to be treated as a thermally fluctuating crystalline membrane..$^{7-10}$ It was shown recently both experimentally ${ }^{11-13}$ and theoretically ${ }^{14}$ that thermal fluctuations lead to a dramatic reduction of the Young modulus and increase of the bending rigidity for micron-sized graphene samples in comparison with atomic scale values. This makes the use of the standard Föppl-von Karman elasticity (FvK) theory for thin plates ${ }^{5} 6$ not directly applicable to graphene and other single atomic layer membranes. This fact is important because the current interpretation of experimental results is based on the FvK theory. In particular, we show that the FvK-derived Schwerin equation, routinely used to derive the Young modulus from indentation experiments ${ }^{11}$ has to be essentially modified for graphene at room temperature and for micron-sized samples.

Elasticity of thin plates

The deflection of a thin plate under transverse point or uniform load is normally well described by the FvK equations. These form a set of two coupled partial differential equations reading:

$\kappa \Delta^{2} h-\left(\frac{\partial^{2} \phi}{\partial y^{2}} \frac{\partial^{2} h}{\partial x^{2}}+\frac{\partial^{2} \phi}{\partial x^{2}} \frac{\partial^{2} h}{\partial y^{2}}-2 \frac{\partial^{2} \phi}{\partial x \partial y} \frac{\partial^{2} h}{\partial x \partial y}\right)=P$,
$\Delta^{2} \phi+Y\left(\frac{\partial^{2} h}{\partial x^{2}} \frac{\partial^{2} h}{\partial y^{2}}-\left(\frac{\partial^{2} h}{\partial x \partial y}\right)^{2}\right)=0$,

where $h$ is the displacement in the direction perpendicular to the plane, i.e., the deflection, $\phi$ is the potential for the in-plane stress tensor, $K$ is the bending rigidity, $Y$ the two-dimensional (2D) Young modulus and $P$ the transverse pressure.

The behavior of $h$ as a function of $P$ and system size $L$ can be obtained from a scaling analysis of the FvK equations as follows. Equation 2 implies that $\phi / L^{4} \sim Y h^{2} / L^{4}$ or $\phi \sim Y h^{2}$. Then the second term in Eq. 1 scales as $\phi h / L^{4} \sim Y h^{3} / L^{4}$ and dominates over the first term $\left(\sim K h / L^{4}\right)$ in the regime of pressures yielding $h^{2}>>K / Y$. For graphene, with $K \simeq 1.1 \mathrm{eV}$ and $Y \simeq 19.6 \mathrm{eV} / \AA$ at room temperature, $^{14,}{ }^{15}$ this condition implies $h>0.23 \AA$ and is normally fulfilled for a system of mesoscopic size (or beyond), except for very low pressure. Hence, apart from this very low pressure regime, the deflection behaves as:

$h \simeq\left(\frac{L^{4} P}{g Y}\right)^{1 / 3}$,

where $g$ is a dimensionless number depending on the shape of the $2 \mathrm{D}$ system and on the type of load, for instance uniform pressure or point load with a tip as in nano-indentation. ${ }^{2}$ In the latter case, the pressure $P$ in Eq. 3 should be replaced by $4 F /\left(\pi L^{2}\right)$ with $F$ the force exerted by the tip. Then, Eq. 3 turns into an equation, which is equivalent to the so-called Schwerin equation, but without prestress. The Schwerin equation is commonly used to determine the elastic modulus $Y$ from nano-indentation measurements. $^{2,} 11$ From now on, we will consider a circular drum of radius $R=L / 2$, clamped at the edge, with $h$ being the midpoint deflection. In that case, the value of $g$ for point load has been derived from analytical solutions of the governing equations and is given by $g=16 /\left(\pi \tilde{g}_{\nu}^{3}\right)$, with $\tilde{g}_{\nu} \simeq 1.0491-0.1462 \nu-$ $0.1583 \nu^{2}$ and $v$ the Poisson ratio. ${ }^{16}$ With $\nu \simeq 0.26$ for graphene, one finds $\tilde{g}_{\nu} \simeq 1.0004$ and $g \simeq 5.087$. For uniform load, a fully

${ }^{1}$ Radboud University, Institute for Molecules and Materials, Heyendaalseweg 135, Nijmegen 6525 AJ, Netherlands

Correspondence: A. Fasolino (a.fasolino@science.ru.nl)

Received: 4 November 2016 Revised: 11 January 2017 Accepted: 27 January 2017

Published online: 22 May 2017 
analytical solution is not available, but there are various approximate, semi-analytical solutions. The solutions reported in refs. 6,16 yield $g_{\nu} \simeq 0.7179-0.1706 \nu-0.1495 \nu^{2} \simeq 0.663$, which is similar to the value obtained in ref. 17 yielding $g_{\nu} \simeq 75\left(1-\nu^{2}\right) /$ $\left(8\left(23+18 \nu-3 \nu^{2}\right)\right) \simeq 0.686$. It was noted, ${ }^{17}$ however, that these expressions underestimate by $10 \%$ the values obtained from the classical, more complex and accurate solution by Hencky ${ }^{18}$ for the case that $v=0.16$. Here, we will use $g_{v}=0.714$ yielding $g \simeq 43.9$. With this larger value for $g_{v}$, compatible with Hencky's solution, the simulation data for $h$ vs. $P$ yield the correct (known) value of $Y$, which agrees with that obtained from a simulation with point load. We will comment on this later on.

At low enough pressure, where $h^{2}<<\kappa / Y$, we have a linear regime where $h \simeq L^{4} P /(f K)$ according to the above scaling analysis with $f$ as another dimensionless, numerical factor. From the work in ref. 6,16 , identifying the bending stiffness $Y_{3 D} d^{3} /$ $\left(12\left(1-\nu^{2}\right)\right)$ for a thin plate with bulk Young modulus $Y_{3 D}$ and thickness $d$ as $k$ for a membrane of atomic thickness, we can evaluate $f \simeq 1024.0$ and $f=256.0$ for uniform and point load, respectively.

Summing up the two terms from the scaling analysis, one finds that $h(P, L)$ satisfies the equation:

$f \kappa h+g Y h^{3}=L^{4} P$,

in agreement with the analysis in refs. 16, 19. If we define the cross-over pressure $P_{c 1}$ as the pressure for which the linear term equals the non-linear term in Eq. 4, which is the case when $h^{2}=f_{K} /$ $(g Y)$, we find that the linear regime vanishes rapidly with system size as $P_{c 1} \simeq 2 \sqrt{(f K)^{3} /(g Y) / L^{4}}$.

The above analysis is based on the assumption that the elastic moduli $K$ and $Y$ are constant, i.e., independent of the system size. Recently it has been clearly confirmed, ${ }^{14}$ however, that the elastic moduli of graphene are not material constants but scale as powerlaws of the system size due to strong anharmonic coupling between in-plane modes and large out-of-plane modes, as predicted by membrane theory. ${ }^{20}$ Besides, the moduli exhibit an anomalously strong dependences on strain. ${ }^{14}$ Thus, generally speaking, for a 2D thermally fluctuating solid, the above analysis is invalid and has to be adapted. Eventually this will lead to an anomalous deflection vs. load relation $h \sim P^{a}$ with $a$ different from $1 / 3$ (Eq. 3), as we will show explicitly below. Besides analytical results based on a revised scaling analysis for membranes, we also present the results from atomistic simulations for a graphene drum under uniform load, to validate our analytical findings.

\section{RESULTS}

Scaling theory

In order to account for the size and strain dependence of the elastic moduli, we extend our scaling analysis by replacing these moduli by their renormalized values $K_{\mathrm{R}}$ and $Y_{\mathrm{R}}$. The latter is given by: ${ }^{9}, 20$

$$
\frac{Y_{\mathrm{R}}}{Y} \sim\left\{\begin{array}{ll}
\left(\frac{L}{L_{G}}\right)^{-\eta_{u}} & L_{G}<L<L_{\sigma} \\
\left(\frac{L_{\sigma}}{L_{G}}\right)^{-\eta_{u}} & L_{G}<L_{\sigma} \leq L
\end{array},\right.
$$

while $Y_{\mathrm{R}} / Y=1$ for $L<L_{\mathrm{G}}$ or $L_{\sigma}<L_{\mathrm{G}}$ with $\eta_{u} \simeq 0.325,{ }^{14}$ where $L_{\mathrm{G}}$ is the so-called Ginzburg length beyond which the power-law scaling is applicable. The length $L_{\sigma}$ is the size beyond which anharmonicity is suppressed due to tensile strain and is given by: ${ }^{21}$

$$
L_{\sigma}=\left(\frac{(2 \pi)^{2} K}{2 B \epsilon L_{G}^{\eta}}\right)^{\frac{1}{2-\eta}}=\left(\frac{(2 \pi)^{2} K}{f_{\nu} Y \epsilon L_{G}^{\eta}}\right)^{\frac{1}{2-\eta}}
$$

where $\epsilon$ is the average strain and where we used the relation $2 B=$
$Y /(1-v)=f_{\nu} Y$ with $f_{\nu} \equiv 1 /(1-\nu) \simeq 1.35$ (see also Supplementary Information S2). An equation similar to Eq. 5 applies to $\kappa_{R} / K_{\text {, but }}$ with $-\eta_{u}$ replaced by $\eta \simeq 1-\eta_{u} / 2 \simeq 0.8375$, implying that $\kappa_{\mathrm{R}}$ increases with size, while $Y_{\mathrm{R}}$ decreases with size.

A theoretical estimate for $L_{G}, L_{G}^{\text {theor }}=2 \pi \sqrt{16 \pi \kappa^{2} /\left(3 Y k_{B} T\right)},{ }^{20}$ yields $L_{\mathrm{G}} \sim 40 \AA$ at room temperature, but from simulations, ${ }^{14} L_{\mathrm{G}}$ turned out to be about a factor 2 smaller. Therefore, in the further analysis, we will use $L_{G}=c_{G} L_{G}^{\text {theor }}$, where $c_{G} \simeq 0.415$ at $300 \mathrm{~K}$ is a correction factor resulting from analysis of the simulation data for $Y_{\mathrm{R}}$ as a function of strain reported in ref.14 (see Supplementary Information S1).

With renormalized elastic constants, Eq. 4 still holds, but with $k$ and $Y$ replaced by $\kappa_{\mathrm{R}}$ and $Y_{\mathrm{R}}$. Then, we can again determine the cross-over pressure $P_{c 1}$ imposing equality of the two terms on the left-hand size. For small load where $L_{\sigma}>L$, applying the first line of Eq. 5, one finds $P_{c 1} \sim L^{-(6-\eta) / 2} \sim L^{-2.58}$. For larger loads yielding $L_{\sigma}<L$, however, $P_{c 1}$ acquires a different size dependence:

$P_{c 1}=\frac{\tilde{g}_{1} K^{\frac{8-8 \eta}{8-4 \eta}} Y^{\frac{3 n-2}{8-4 \eta}}\left(k_{\mathrm{B}} T\right)^{\frac{2+\eta}{8-4 \eta}}}{L^{\frac{14-9 \eta}{4-2 \eta}}}$,

where $\quad(14-9 \eta) /(4-2 \eta) \simeq 2.78$ and $\quad \tilde{g}_{1} \simeq 0.55 f^{\nu_{1}} g^{\nu_{2}}$ $\left(c_{G}^{2} g_{\sigma} g_{\epsilon}\right)^{-\nu_{3}}$, with $v_{1}=(10-7 \eta) /(8-4 \eta), v_{2}=(3 \eta-2) /(8-4 \eta)$ and $v_{3}=(2+\eta) /(8-4 \eta)$. The cross-over in the size dependence of $P_{c 1}$ at a system size $L_{c 1}$ should occur at another critical pressure, $P_{c 2}$ where $L_{\sigma}$ is equal to $L_{c 1}$. Explicit expressions for $P_{c 2}$ as a function of $L$ will be given below. For graphene, it turns out that $L_{c 1}$ would be smaller than $L_{G}$, thus in a regime where renormalization does not apply. For $L>L_{\mathrm{G}}>L_{c 1}, P_{c 2}<P_{c 1}$, implying that $L_{\sigma}<L$ at $P_{c 1}$. Therefore, for graphene $P_{c 1}$ is always given by Eq. 7, derived from the second line of Eq. 5, and has no cross-over. Thus, with renormalized elastic moduli, the regime where the first term in Eq. 4 is dominant still vanishes for $L \rightarrow \infty$ but more slowly, namely as $P_{c 1} \sim L^{-2.78}$ instead of $L^{-4}$.

A third critical pressure $P_{c 3}$ is defined as the pressure for which anharmonicity is completely suppressed, i.e., where $L_{\sigma} \leq L_{G}$ yielding $Y_{R}=Y$. The important observation to make now is that for pressures $P$ within $P_{c 2}<P<P_{c 3}$ or equivalently $L_{G}<L_{\sigma}<L, h$ as a function of $P$ obeys a power law different from that in Eq. 3 , due to the renormalization of the elastic moduli. Indeed, using Eqs. 5 and $6, Y_{\mathrm{R}}$ depends on the strain $\epsilon$ as:

$Y_{\mathrm{R}}(\epsilon) \simeq Y\left(\frac{(2 \pi)^{2} K}{f_{\nu} Y \epsilon L_{G}^{2}}\right)^{-\mu} \simeq Y\left(\frac{16 \pi \kappa c_{G}^{2} f_{\nu} \epsilon}{3 k_{B} T}\right)^{\mu}$,

with $\mu \equiv(2-2 \eta) /(2-\eta) \simeq 0.2797$. In a similar way one can derive an expression for $\kappa_{\mathrm{R}}(\epsilon)$. Substitution of Eq. 8 with $\epsilon \simeq$ $g_{\epsilon} h^{2} / L^{2}$ into Eq. 3 with $Y$ replaced by $Y_{\mathrm{R}}$ gives a self-consistency equation for $h$ with solution:

$h \simeq\left(\frac{k_{\mathrm{B}} T}{K}\right)^{\frac{\mu}{3+2 \mu}}\left(\frac{L^{4+2 \mu} P}{\tilde{g} Y}\right)^{\frac{1}{3+2 \mu}}$

where $(3+2 \mu) \simeq 3.56$ and $\tilde{g} \simeq\left(16 \pi c_{G}^{2} f_{\nu} g_{\epsilon} / 3\right)^{\mu} g$. This equation replaces Eq. 3 for the case of a $2 \mathrm{D}$ solid exhibiting renormalization of the elastic moduli according to membrane theory. One should notice that now the relation between $h$ and $P$ involves, apart from $Y$, also $k_{\mathrm{B}} T / K$, which is natural as this quantity controls the strength of anharmonic coupling. Notice that Eq. 3 is recovered from Eq. 9 for $\mu=0$ (i.e., for $\eta_{u}=0$ ).

The geometrical prefactor $g_{\epsilon}$ for the strain in $\epsilon=g_{\epsilon} h^{2} / L^{2}$ depends on the shape of the deflected membrane. If we define $\epsilon=\sqrt{A / A_{0}}-1$ with $A$ the surface area of the deflected membrane and $A_{0}=\pi L^{2} / 4$ that of the flat drum, then for uniform load, with the shape of the drum approximately being that of a spherical cap, $g_{\epsilon}=2$, whereas for nano-indentation, with an approximately cone shaped membrane, $g_{\epsilon}=1$.

To find the critical pressure $P_{c 2}$, we first need to solve $L_{\sigma}(h)=L$ for $h$. Substitution of this $h$ into Eq. 4, retaining both terms on the 
left-hand side with $\kappa$ and $Y$ replaced by $\kappa_{R}$ and $Y_{R}$, respectively leads to:

$P_{c 2}=\frac{g_{21}\left(k_{\mathrm{B}} T\right)^{\frac{3 n}{4}} K^{\frac{3-3 \eta}{2}} Y^{\frac{3 n-2}{4}}}{L^{\frac{8-3 n}{2}}}+\frac{g_{22}\left(k_{\mathrm{B}} T\right)^{\frac{7 \eta-4}{4}} K^{\frac{7-7 n}{2}}}{Y^{\frac{6-7 \eta}{4}} L^{\frac{12-7 \eta}{2}}}$

with $(8-3 \eta) / 2 \simeq 2.74,(12-7 \eta) / 2 \simeq 3.07, g_{21} \simeq 0.1063 f\left(c_{\mathrm{G}}^{3 \eta}\right.$ $\left.f_{\nu} g_{\epsilon}\right)^{-1 / 2}$ and $g_{22} \simeq 12.06 g\left(c_{\mathrm{G}}^{7 \eta-4} f_{\nu}^{3} g_{\epsilon}^{3}\right)^{-1 / 2}$.

In a similar way, for deriving $P_{c 3}$, we first solve $L_{\sigma}(h)=L_{\mathrm{G}}$ for $h$ and then substitute this $h$ into Eq. 4 . In this pressure regime, the contribution from the term with $\kappa$ is very small (for $L$ not too small) and can be neglected. Then, we obtain:

$P_{c 3}=\frac{g_{3} Y}{L}\left(\frac{k_{\mathrm{B}} T}{K}\right)^{3 / 2}$

with $g_{3} \simeq 0.0146 g\left(c_{\mathrm{G}}^{2} f_{\nu} g_{\epsilon}\right)^{-3 / 2}$. If we just consider the pressure contribution $\rho m g$ due to the mass of the carbon atoms, with $\rho$ the 2D density of graphene and $g$ the gravitational acceleration, it can directly be calculated from Eq. 11 that it requires a system size of about $L=305 \mathrm{~km}$ (!) to suppress anharmonicities by graphene's own weight.

The behavior for the various critical loads as a function of system size is depicted in Fig. 1 on two different length scales, corresponding to the scale used in our simulations and the typical scale in experiments, respectively. For the latter case, we used the parameters for point load and displayed critical forces $F_{c i}=$ $\pi L^{2} P_{c i} / 4(i=1,2,3)$ instead of pressures.

\section{Atomistic simulations}

In order to validate the behavior derived above and in particular Eq. 9, we have performed atomistic, Monte Carlo (MC) simulations for a graphene drum with a diameter of $L \simeq 315 \AA$ under uniform

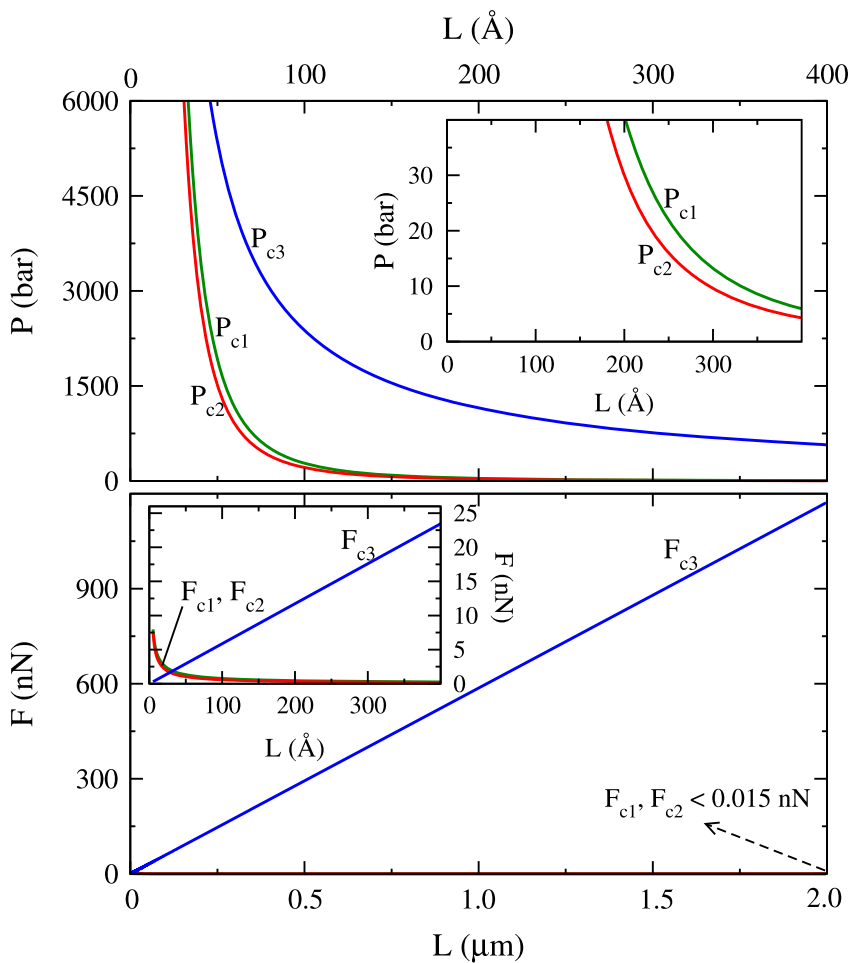

Fig. 1 Calculated critical pressures $P_{\mathrm{c} 1}, P_{\mathrm{c} 2}, P_{\mathrm{c} 3}$ according to Eqs. 7, 10, 11 for uniform load (top panels) and critical forces $F_{\mathrm{c} 1}, F_{\mathrm{c} 2}, F_{\mathrm{c} 3}$ for point load (bottom panels) as a function of system size $L$ on two different scales, in the absence of prestrain. The inset zoom in at different load and/or size range
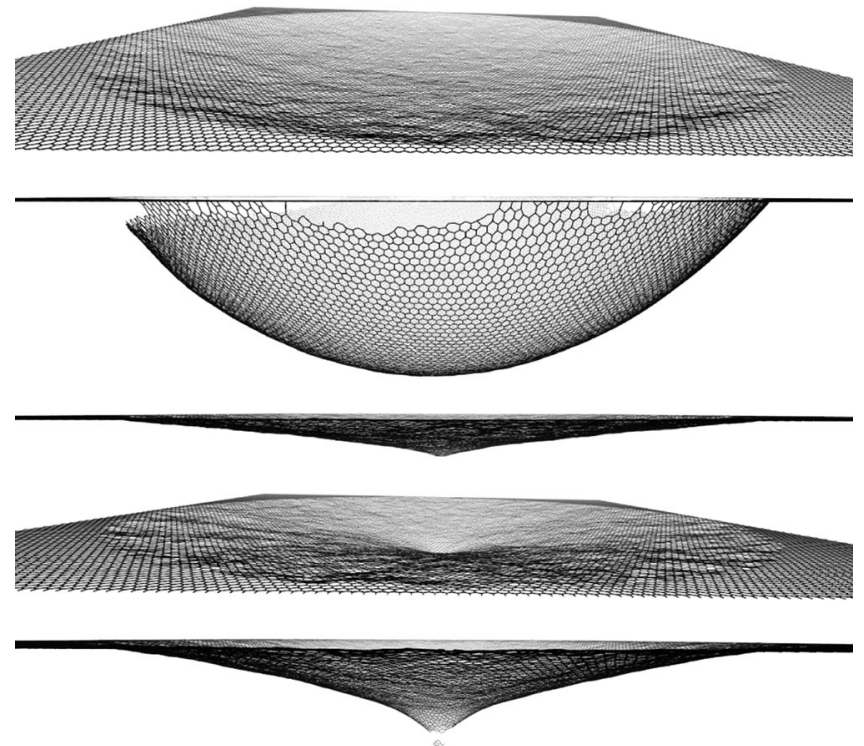

Fig. 2 Snapshots from simulations of the indentation of a graphene drum with diameter $L \simeq 315 \AA$ under uniform load (upper three graphs) and point load (lower three graphs). In the graphs with a view at an angle, one can see the thermal corrugation of the drum, responsible for the anharmonic effects. The bottom graphs show configurations just after breaking. For uniform load breaking occurs at the drum edge at $P \simeq 60 \mathrm{kbar}$, while for point load it occurs around the tip at $F \simeq 160 \mathrm{nN}$ for the given system size

transverse pressures over a wide range between 0 and 60 kbar. The LCBOPII model was used for the carbon interatomic interactions $\mathrm{s}^{22}$ and the pressure was modelled by assigning a weight $M=P /(\rho g)$ to each atom. Defining the $z$-direction perpendicular to the drum, a change $\delta z$ of the $z$-coordinate of an atom contributes an amount $M g \delta z$ to the energy change $\Delta E$ of the system entering the $\mathrm{MC}$ acceptance probability $P_{\mathrm{acc}}=\min [1$, $\left.\exp \left(-\Delta E / k_{\mathrm{B}} T\right)\right]$ for the configurational change. The simulations were performed at $T=300 \mathrm{~K}$ and the $2 \mathrm{D}$ density of the drum was adjusted to the equilibrium density at $300 \mathrm{~K}$, so that no prestrain was present. For illustration, snapshots from these simulations are shown in Fig. 2, together with snapshots from a simulation under point load. In the latter case, only atoms in a small circular, central region were assigned a weight $M=F /\left(N_{c} g\right)$, with $F$ the total applied force and $N_{c}$ the number of atoms in the central circle. For our simulation, $N_{c}=25$.

The simulation results for uniform pressure $P$ vs. $h$ are given in Fig. 3. Before analyzing these data, we should realize that for the derivation of Eq. 9, we have tacitly neglected the linear term in Eq. 4, which is justified for system sizes commonly used in experiments of the order of $1 \mu \mathrm{m}$ or larger, yielding $P_{c 1}<0.003 \mathrm{bar}$. The system size $L \simeq 315 \AA$ used in our simulations, however, requires to include the linear regime to cover the pressure range smaller then $P_{c 1} \simeq 12$ bar in this case. While for $P<P_{c 2}, P$ as a function of $h$ should behave as $P=A h+B h^{3}$; for $P>P$, the expected behavior is $P=A h^{(2-3 \eta) /(2-\eta)}+B h^{3+2 \mu} \simeq A h^{-0.441}+$ $B h^{3.559}$. In order to fit our simulation data for pressures below and above $P_{c 2}$, we used the following form, which combines the usual FvK linear term with the renormalized expressions yielding correct asymptotic behaviour for $h \rightarrow 0$ and $h \rightarrow \infty$ :

$P=A h+B h^{3+2 \mu}$

It has two fitting parameters $A$ and $B$ of which the latter is related to the elastic moduli by $B=\left(\kappa / k_{\mathrm{B}} T\right)^{\mu} \tilde{g} Y / L^{4+2 \mu}$. 


\section{4}

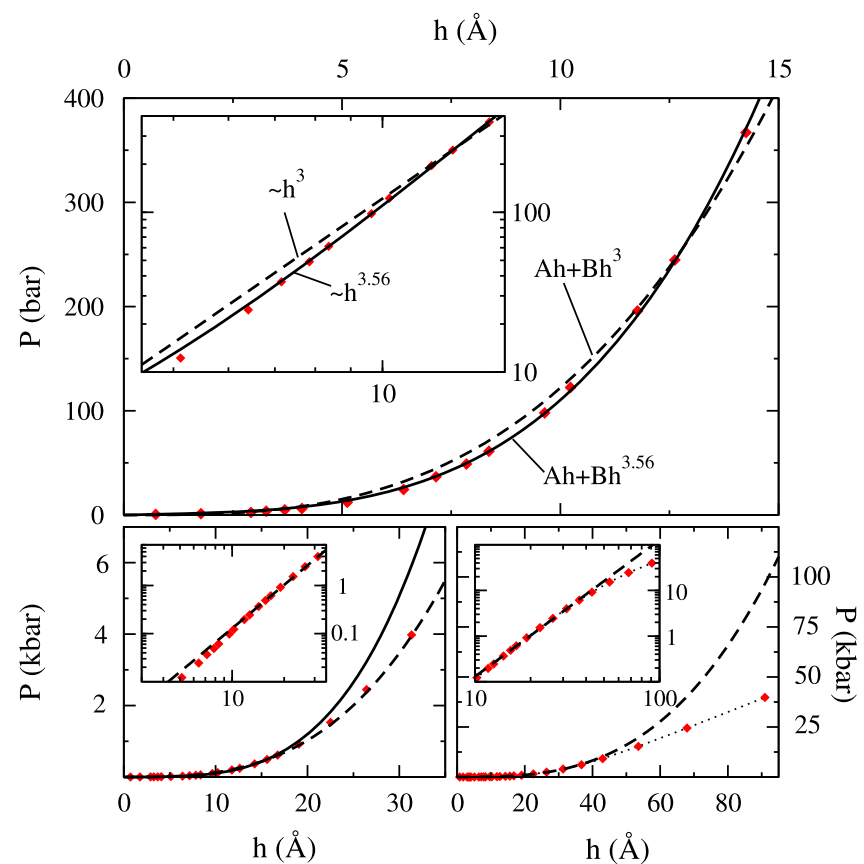

Fig. 3 Pressure as a function of the midpoint deflection obtained from simulations (symbols) on three different deflection ranges. The solid line is a best fit according to Eq. 12 over the interval $h \in[0,15] \AA$, while the dashed lines are best fits according $P=A h+B h^{3}$ over the interval $h \in[0,15] \AA$ (upper panel) and $h \in[15,35] \AA$ (lower panels), respectively. These insets are the same plots in log-log scale

The upper panel in Fig. 3 shows that, for pressures up to $\sim 400$ bar, the simulation data are in good agreement with Eq. 12, as shown by the best fit (solid line), and clearly deviate from a best fit based on $P=A h+B h^{3}$, expected without renormalization of the elastic constants. Instead, beyond 400 bar up to about 6000 bar shown in the left bottom panel, the data points are best fitted by $P=A h+B h^{3} \simeq B h^{3}$, which according to our analysis should apply for $P>P_{c 3}$. This suggests that $P_{c 3} \simeq 400$ bar, about a factor 2 smaller than the estimate from Eq. 11 (see also Fig. 1) but nevertheless of the right order of magnitude. The numerical discrepancy here might be due to the fact that cross-over regimes are ignored in the theoretical derivations.

The best fit for pressures in the range $[400-40,000]$ bar, beyond $P_{c 3}$, based on $P=A h+B h^{3}$ yields $B \simeq 0.128$, implying a (bare) Young elastic modulus $Y=L^{4} B / g \simeq 299 \mathrm{~N} / \mathrm{m}$. This is the value after the mentioned adjustment of $g_{v}$ such that it was equal to the $Y$ obtained from a point load simulation at an applied force beyond $F_{c 3}$. The found value is close to the known true bare modulus $Y=314 \mathrm{~N} / \mathrm{m}$ at $300 \mathrm{~K}$ for LCBOPII. From the best fit at low pressures $\left(<P_{c 3}\right)$ based on Eq. 12 and with $K=1.1 \mathrm{eV}$, we obtain $Y$ $=307 \mathrm{~N} / \mathrm{m}$. The small difference with the value from the first method may be due an uncertainty in the factor $c_{g}$. In fact, the above two ways to determine $Y$ (for known $\kappa$ ) can alternatively be used to determine $\tilde{g}$ and $c_{\mathrm{G}}$ (and $L_{\mathrm{G}}$ ) by imposing equality of $Y$.

\section{DISCUSSION}

A way to extract the renormalization of $Y$ from the simulation data is to make a fit based on Eq. 12 and then use that $B h^{3+2 \mu}=$ $Y_{\mathrm{R}} h^{3} /\left(g L^{4}\right)$ to obtain the strain dependent $Y_{\mathrm{R}}$ :

$Y_{\mathrm{R}}(\epsilon)=\frac{L^{4+2 \mu} B}{g}\left(\frac{h^{2}}{L^{2}}\right)^{\mu}=\frac{L^{4+2 \mu} B}{g g_{\varepsilon}^{\mu}} \epsilon^{\mu}$,

valid for $\epsilon<\epsilon_{c 3}$, where $\epsilon_{c 3} \simeq 0.005$ is the strain required to suppress anharmonicity, i.e., the strain beyond which the normal $P \sim h^{3}$ is applicable. Notice that this approach does not require knowledge of $\kappa$ nor $\tilde{g}$. For point load, the same approach can be used, but with an additional factor $\pi /\left(4 L^{2}\right)$ multiplying the right-hand side of Eq. 13.

The right bottom panel of Fig. 3 shows a deviation from the $P \sim$ $h^{3}$ behavior for deflections beyond $40 \AA$. This deviation for large strain can be attributed to a normal softening of the elastic moduli due to stretching anharmonicity, as in 3D crystals. Such a softening for graphene under large strain is in agreement with previous observations. ${ }^{23}$ The corresponding critical pressure, $P_{c 4}$, should depend on the size as $P_{c 4}=g_{4} / L$, with $g_{4} \simeq 25.2 \mathrm{~N} / \mathrm{m}$ derived from the value $P_{c 4} \simeq 8 \mathrm{kbar}\left(8 \times 10^{8} \mathrm{~N}\right)$ for $L=3.15 \times 10^{-8}$ $\mathrm{m}$. Indeed, assuming that this anharmonicity sets in at a fixed (size independent) critical strain, $\epsilon_{c 4}=g_{\epsilon} h_{c 4}^{2} / L^{2}$, the size dependence of $P_{c 4}$ follows directly from Eq. 4, neglecting the linear term, yielding $P_{c 4} \simeq(g Y / L)\left(\epsilon_{c 4} / g_{\epsilon}\right)^{3 / 2}$, with $\epsilon_{c 4} \simeq 0.032$. Although $P_{c 4}$ is a safe lower bound for breaking, normally breaking is only expected at significantly higher strains, where the in-plane moduli start to vanish due to the anharmonicity of the interaction potential. For LCBOPII, the bulk modulus vanishes at a strain value of $\sim 0.2$, a value indeed close to the strain where breaking was actually observed in our simulation, at a pressure $P_{\mathrm{br}} \simeq 50 \mathrm{kbar}$. This value of the breaking strain is similar to that found in a simulation study of graphene nanoribbons under uniaxial strain. ${ }^{24}$ It should be noticed, however, that a typical atomistic simulation only covers a very small time interval, typically orders of magnitude smaller than a second, which makes the choice of a maximal, safe lower bound for breaking from simulations at a given temperature not obvious and somewhat arbitrary.

Staying on the safe side by choosing the breaking pressure as $P_{\mathrm{br}}=4 P_{\mathrm{c} 4}=4 g_{4} / L$, yielding $P_{\mathrm{br}} \simeq 32 \mathrm{kbar}$ for the simulated system size with a corresponding strain of $\sim 0.13$, a graphene drum of $1 \mathrm{~m}$ in diameter gives a breaking force of $F_{c 4}=\pi L^{2} P_{c 4} / 4 \simeq 79.2 \mathrm{~N}$, enough for an extremely heavy cat of about $8.0 \mathrm{~kg}$ to be safe, treating it as a uniform load. Treating the cat as a point load, however, and assuming that the breaking strain is equal to that for uniform load, we have to correct $g_{4}$ by a factor $\sim 0.328$ due to the different values for $g$ and $g_{\epsilon}$, implying that the cat should not be heavier than $2.65 \mathrm{~kg}$, i.e., a young cat, to be safe. In reality, a cat on a drum of this size is something between point and uniform load, so that probably any cat should be safe on it. It is interesting to notice, and somewhat counterintuitive, that while a drum of $1 \mathrm{~m}$ cannot bear a person of $100 \mathrm{~kg}$, a drum of $40 \mathrm{~m}$ could, due to the fact that $F_{c 4}$ grows linearly with $L$. A graphene drum would only break by its own weight for a size $L=4 g_{4} /(\rho m g) \simeq 13520 \mathrm{~km}$ !

While the relations derived above are appropriate for the analysis of our simulations where prestress can be controlled and taken to be zero, it should be noticed that in nano-indentation experiments, almost unavoidably some prestress $\sigma_{0}$ is present, created during preparation of the drum. The implications for the load vs. deflection expression and the various critical loads for the case of tensile prestress, including renormalization of the elastic moduli, are given in the Supplementary Information S3. Tensile prestress gives rise to a contribution to the force, which is linear in $h$, namely $\pi \sigma_{0} h$. As this is an order $L^{2}$ larger then the linear contribution $K h / L^{2}$ arising from the FvK equations, its contribution can be significant as compared to the cubic term $Y h^{3} / L^{2}$, even for $\mu \mathrm{m}$-sized drums. Therefore, for the sake of accuracy in measuring the elastic modulus and the effect of its renormalization, one should keep the prestress as small as possible, so that the term cubic in $h$, from which the elastic modulus is determined, is the dominant term. Moreover, while $F_{c 1}$ increases with system size for tensile prestress, $F_{c 2}$ and $F_{c 3}$ decrease with $\sigma_{0}$. To be able to measure the renormalization of the elastic modulus, however, $F_{c 3}$ should not be too small, leaving a sufficiently large force domain $\left(<F_{c 3}\right)$ for observing renormalization. 


\section{CONCLUSIONS}

The revised FvK theory for thermally excited membranes like graphene that we have presented here is important for any technological application of 2D materials involving their mechanical properties. For graphene, we have discussed in a quantitative way the behaviour under uniform and point load up to breaking.

\section{CHANGE HISTORY}

A correction to this article has been published and is linked from the HTML version of this article.

\section{ACKNOWLEDGEMENTS}

This project has received funding from the European Unions Horizon 2020 research and innovation programme under grant agreement No. 696656 GrapheneCore1. We thank Cristina Gomez-Navarro, Julio Gomez-Herrero and Guillermo López-Polín for interesting discussion.

\section{COMPETING INTERESTS}

The authors declare no competing financial interests.

\section{REFERENCES}

1. Booth, T. J. et al. Macroscopic graphene membranes and their extraordinary stiffness. Nano Lett. 8, 2442-2446 (2008).

2. Lee, C., Wei, X., Kysar, J. W. \& Hone, J. Measurement of the elastic properties and intrinsic strength of monolayer graphene. Science 321, 385-388 (2008).

3. Vickery, J. L., Patil, A. J. \& Mann, S. Fabrication of graphene polymer nanocomposites with higher-order three-dimensional architectures. Adv. Mater. 21, 2180-2184 (2009).

4. Dolleman, R. J., Davidovikj, D., Cartamil-Bueno, S. J., van der Zant, H. S. J. \& Steeneken, P. G. Graphene squeeze-film pressure sensors. Nano Lett. 16, 568 (2016).

5. Landau, L. D. \& Lifshitz, E. M. Theory of Elasticity. (Pergamon: Oxford, 1970).

6. Timoshenko, S. P. \& Woinowsky-Krieger, S. Theory of Plates and Shells. (McGrawHill, 1951).

7. Katsnelson, M. I. Graphene: Carbon in Two Dimensions. (Cambridge University Press: Cambrigde, 2012).

8. Katsnelson, M. I. \& Fasolino, A. Graphene as a prototype crystalline membrane. Acc. Chem. Res. 46, 97-105 (2013).

9. Kosmrlj, A. \& Nelson, D. R. Response of thermalized ribbons to pulling and bending. Phys. Rev. B 93, 125431 (2016).

10. Gornyi, I. V., Kachorovskii, V. Yu. \& Mirlin, A. D., Anomalous Hooke's law in disordered graphene. Preprint at arXiv:1603.00398.
11. López-Polín, G. et al. Increasing the elastic modulus of graphene by controlled defect creation. Nat. Phys. 11, 26-31 (2015).

12. Blees, M. K. et al. Graphene kirigami. Nature 524, 204-207 (2015).

13. Nicholl, R. J. T. et al. Mechanics of free-standing graphene: stretching a crumpled membrane. Nat. Commun. 6, 8789 (2015).

14. Los, J. H., Fasolino, A. \& Katsnelson, M. I. Scaling behavior and strain dependence of in-plane elastic properties of graphene. Phys. Rev. Lett. 116, 015901 (2016).

15. Fasolino, A., Los, J. H. \& Katsnelson, M. I. Intrinsic ripples in graphene. Nat. Mater. 6, 858-861 (2007).

16. Komaragiri, U. \& Begley, M. R. The mechanical response of freestanding circular elastic films under point and pressure loads. J. Appl. Mech. 72, 203 (2005).

17. Yue K., Gao W., Huang R. \& Liechti K. M. Analytical methods for the mechanics of graphene bubbles. J. Appl. Phys. 112, 083512 (2012).

18. Hencky, H. Über den Spannungszustand in kreisrunden Platten mit verschwindender Biegungssteifigkeit. Z. Math. Phys. 63, 311 (1915).

19. Wang, P., Gao, W., Zhiyi Cao, Z., Liechti, K. M. \& Huang, R. Numerical analysis of circular graphene bubbles J. Appl. Mech. 80, 040905 (2013).

20. Nelson, D. R., Piran, T., \& Weinberg, S. (eds) Statistical Mechanics of Membranes and Surfaces (World Scientific, 2004).

21. Roldán, R., Fasolino, A., Zakharchenko, K. V. \& Katsnelson, M. I. Suppression of anharmonicities in crystalline membranes by external strain. Phys. Rev. B $\mathbf{8 3}$ 174104 (2011).

22. Los, J. H., Ghiringhelli, L. M., Meijer, E. J. \& Fasolino, A. Improved long-range reactive bond-order potential for carbon. I. Construction. Phys. Rev. B 72, 214102 (2005).

23. Gao W., Huang R. Thermomechanics of monolayer graphene: Rippling, thermal expansion and elasticity. J. Mech. Phys. Solids 66, 42 (2014).

24. Lu, Q., Gao, W. \& Huang, R. Atomistic simulation and continuum modeling of graphene nanoribbons under uniaxial tension. Modelling Simul. Mater. Sci. Eng. 19, 054006 (2011).

\begin{abstract}
Access This article is licensed under a Creative Commons Attribution 4.0 International License, which permits use, sharing, adaptation, distribution and reproduction in any medium or format, as long as you give appropriate credit to the original author(s) and the source, provide a link to the Creative Commons license, and indicate if changes were made. The images or other third party material in this article are included in the article's Creative Commons license, unless indicated otherwise in a credit line to the material. If material is not included in the article's Creative Commons license and your intended use is not permitted by statutory regulation or exceeds the permitted use, you will need to obtain permission directly from the copyright holder. To view a copy of this license, visit http://creativecommons. org/licenses/by/4.0/.
\end{abstract}

(c) The Author(s) 2017

Supplementary Information accompanies the paper on the npj 2D Materials and Applications website (doi:10.1038/s41699-017-0009-3). 